\title{
The theory of nonlinear systems as an instrument for solving engineering problems
}

\author{
Olga I. Ohrimenko ${ }^{1 *}$, Igor M. Maltsev ${ }^{1}$, Violetta . Rokotyanskaya ${ }^{2}$, Maria L. Vilisova ${ }^{1}$ \\ ${ }^{1}$ Don State Technical University, 344002 Rostov-on-Don, Russian Federation \\ ${ }^{2}$ Russian State Agrarian University named after K.A. Timiryazev, 127550 Moscow, Russia
}

\begin{abstract}
The article outlines theoretical, methodological and practical issues of modern control and optimization theory, as well as the problems of nonlinear systems theory. Theoretical conclusions and results allowed to build mathematical models applicable to the management of objects of different nature with different principles of action, in particular, to the management of complex technical and technological objects that can be considered as nonlinear dynamic systems. The authors find it appropriate to consider nonlinear dynamic integral models as Volterra integro-power series from many functional arguments with multidimensional weight functions and a certain finite set of inputs to the system. The set of multidimensional kernels of integral Volterra operators completely characterizes the nonlinear and dynamic properties, and, consequently, the technical state of the initial system. The application of Volterra series based models allows to take into account the nonlinear and inertial properties of the initial nonlinear dynamic system more fully and accurately, it also makes the model diagnostic of a technical system more universal, raises the reliability of the forecast. The diagnostic procedure in this case is aimed at defining Volterra kernels based on the data of "input-output" experiment and building the diagnostic system of attribute in the space of which the decisive rule of optimal classification is created.
\end{abstract}

\section{Introduction}

Dynamic controlled processes occur in living organisms, economic and organizational manmachine systems. When these are ignored or overridden, the result can be large losses. The development and complication of systems has led to the creation of automatic control systems (ACS) of technological processes, production and industries [1, 2].

Such systems are closely related, although their functions and technical means, as well as the character of solved tasks differ significantly.

The main problem of optimal dynamic synthesis of technical systems consists in finding specific parameters values ensuring the efficiency of systems' functioning. This is especially

\footnotetext{
${ }^{*}$ Corresponding author: oxrimenko@list.ru
} 
important when creating and studying new system's properties, since all the parameters are critically important for these properties realization $[1,2,3]$.

\section{Methods}

There are many approaches to the methodology of studying different systems and to their definition. In general, a system is a collection of interdependent elements with certain properties, forming a certain integrity.

The choice of this or that way of describing the state-space and external influences as the external environment of the system has particular importance.

Complexity is one of the basic properties of any system that can be used only when comparing with a reference systems, in terms of qualitative and quantitative assessment. At the present stage of system analysis development there is no strict mathematical definition of a complex system covering all intuitive notions of real systems $[5,6,7]$.

A complex system is a system possessing at least one of the following properties:

1) a large number of variables and a large number of connections between them and other parts of the system,

2) a high order of the equations defining the system,

3) a large number of nonlinear elements in the system.

A complex systems' parameters often have a non-numerical nature, which results in a significant dependence of their qualitative analysis and synthesis on the input metrics and topologies in the relevant deterministic environment. The researchers classify such complex systems into multidimensional, multiconnected, multicomponent, highly complex, large, multilevel ones $[3,5,6]$.

Let us briefly overview the systems, characterized by different degrees of complexity:

1. Conservative - systems in which there is at least one element that does not change when the system is exposed to external factors. Such systems are structurally unstable, one of their characteristic features is the existence of an equilibrium point.

2. Dissipative systems are those in which irreversible processes can occur.

3. Systems with equilibrium and nonequilibrium constraints, in which parameters can be fixed at a constant level or may have several stable states.

4. Systems with linear behavior are the simplest ones. However, some additional complexity is due to the backward linkages in them.

When a system is non-linear, or it becomes so in some area, one of the most important factors of complexity, called ambiguity, arises.

5. Isolated systems with an inevitable move to statics. The transition of this system to a state of equilibrium is here equated with the stability of the system.

6. Dynamical systems are changing over time. The major increase in complexity is due to the system's convergence toward non-equilibrium steady state. Their complexity also increases, if the processes occurring in the system are of an oscillatory nature. Among all the dynamical systems, the discrete dynamical systems are the most complex.

7. Systems with algorithmic recording of information.

\section{Results}

The modern phase of technologies development is characterized by a significant increase in the complexity of studied and designed technical systems, as the range of technological operations and constructive solutions becomes wider both in technical objects themselves and their individual assembly units, which leads to an increase in dynamic loads on their structural and functional parts $[4,7,8]$. 
This involves the increased importance of technological processes, characterized by the nonlinear change in various parameters of the system. Thus, it becomes necessary to use the theory of nonlinear systems as an instrument for studying and modeling such systems. The problems of synthesizing nonlinear dynamical systems stays in the focus of attention of applied scientific research for several decades. This is due to a constant search for an adequate theoretical apparatus for the synthesis of linear and nonlinear systems.

In some cases, important for applications, it is appropriate to consider nonlinear dynamic integral models in the form of Volterra integro-power series from many functional arguments $x_{1}(t), x_{2}(t), \ldots, x_{n}(t)$ as follows $[8]$ :

$$
y_{j}(t)=\sum_{n=1}^{\infty} \sum_{i_{1}=1}^{s} \ldots \sum_{i_{n}=1}^{s} \int_{0}^{t} \ldots \int_{0}^{t} w_{i_{1} i_{2} \ldots i_{n}}^{j}\left(\tau_{1}, \tau_{2}, \ldots, \tau_{n}\right) \sum_{k=1}^{n} u_{i_{k}}\left(t-\tau_{k}\right) d \tau_{k},
$$

here $w_{i_{1} i_{2} \ldots i_{n}}^{j}\left(\tau_{1}, \tau_{2}, \ldots, \tau_{n}\right)$ are multidimensional weight functions (so-called Volterra kernels) of $n$-th order by $\left(i_{1}, i_{2}, \ldots, i_{n}\right)$ inputs and $j$-th output, symmetric with respect to $\tau_{1}, \tau_{2}, \ldots, t_{n} ; u(t)-$ input action, and $y(t)$ - output at zero initial condition $y(t)$.

Here, the set of multidimensional kernels of integral Volterra type operators fully characterizes both nonlinear and dynamic properties, and, consequently, the technical condition of the original system.

The application of Volterra series based models allows to take into account more fully and accurately the nonlinear and inertial properties of the original nonlinear dynamic system, makes the modeling diagnostics of a technical system more universal, and increases the reliability of the forecast.

The diagnostic procedure in this case is reduced to the determination of Volterra nuclei according to the input - output experiment and the construction of the diagnostic system of features based on the obtained nuclei, in the space of which the decisive rule of optimal classification is built.

The Volterra integral operator can have the following form:

$$
y(x(t))=\sum_{i=1}^{n} \int_{-T}^{T} \ldots \int_{-T}^{T} h_{i}\left(\tau_{1}, \tau_{2}, \ldots, \tau_{i}\right) x\left(t-\tau_{1}\right) x\left(t-\tau_{2}\right) \ldots x\left(t-\tau_{i}\right) d \tau_{1} d \tau_{2} \ldots d \tau_{i} .
$$

Here, the initial nonlinear dynamic system has the following Volterra kernels: $h_{i}\left(\tau_{1}, \tau_{2}, \ldots, \tau_{i}\right), i=1,2, \ldots, n$.

The corresponding multivariate polynomial Hammerstein filter meeting the criterion of standard mean square filtering error, the mathematical expectation of which $(\mathrm{M})$ is equal to:

$$
M\left\{\left[f\left(u_{1}, u_{2}, \ldots, u_{m}\right)-F\left(h\left(u_{1}, u_{2}, \ldots, u_{m}\right)\right)\right]^{2}\right\},
$$

can be written as:

$$
F\left[h_{1}\left(u_{1}, u_{2}, \ldots, u_{m}\right)\right]=\sum_{k=1}^{n} \int_{0}^{R_{x_{1}}} \int_{0}^{R_{x_{2}}} \ldots \int_{0}^{R_{x_{m}}} \cdot V_{k}\left(x_{1}, x_{2}, \ldots, x_{m}\right) h^{k}\left(u_{1}-x_{1}, u_{2}-x_{2}, \ldots, u_{m}-x_{m}\right) d x_{1} \cdot d x_{2} \ldots d x_{m},
$$

where $V_{k}\left(x_{1}, x_{2}, \ldots, x_{m}\right), k=\overrightarrow{1, n}$ are unknown kernels of the Hammerstein integral operator, that we should define; $R_{x_{1}}, R_{x_{2}}, \ldots, R_{x_{m}}$ are given values of the operator filter memory by coordinates $x_{1}, x_{2}, \ldots, x_{m}$ thus $R_{x_{j}} \in X_{j}, j=\overrightarrow{1, m}$, the point $\left(u_{1}, u_{2}, \ldots, u_{m}\right) \in X_{1} \times X_{2} \times \ldots \times X_{m}$ (the assumed field). 
Finally, for the given pair of functional spaces $E_{1}$ and $E_{2}$ different in topological structure, an optimal estimate of the regularization parameter is obtained [8]. As is known, the random signal $x(t)$ at the input of the system is an additive mixture of the useful signal $\mathrm{s}(\mathrm{t})$ and interference $\mathrm{n}(\mathrm{t})$ :

$$
x(t)=s(t)+n(t),
$$

where the random functions $\mathrm{s}(\mathrm{t})$ and $\mathrm{n}(\mathrm{t})$ are stationary in a narrow sense and are permanently connected. It is required, at measuring the signal $\mathrm{x}(\mathrm{t})$, to carry out the transformation of the useful signal s ( $\mathrm{t})$, given by the function $\Phi[\cdot]$ :

$$
z(t)=\Phi[s(t)] .
$$

In other words, we need to define such transformation $F[\cdot]$ of signal $x(t)$ :

$$
y(t)=F[x(t)]
$$

at which stochastic processes $z(t)$ and $y(t)$ would be close by some criterion. As such a criterion, we used the minimum standard deviation of the signal y from the signal $\mathrm{z}$, that is:

$$
M\left\{[y(t)-z(t)]^{2}\right\}=M\left\{(F[x(t)]-\hat{O}[\mathrm{~s}(t)])^{2}\right\} \rightarrow \min .
$$

The problem consists in isolating the useful signal $\mathrm{s}(\mathrm{t})$ from its additive mixture with the interference $n(t)$ by the criterion of the minimum mean square error

$$
M\left\{(F[x(t)]-\mathrm{s}(t))^{2}\right\} \rightarrow \min .
$$

It is appropriate to formulate the problem of optimal signal transformation (7) as a problem of minimization in space by the function $F, \Phi$, defined on a set of realizations of a stochastic process.

First, we considered the direct product $\mathrm{Z}=\mathrm{X}$ 'S of the spaces $\mathrm{X}, \mathrm{S}$ of stochastic processes $\mathrm{x}(\mathrm{t})$ and $\mathrm{s}(\mathrm{t})$ realizations.

Assuming as known the joint probability density

$$
p_{x, s, n, m}\left(x_{1}, \ldots, x_{n}, y_{1}, \ldots, y_{m}, t_{1}, \ldots, t_{n}, \tau_{1}, \ldots, \tau_{m}\right),
$$

the probability measure was constructed in the space $\mathrm{Z}$.

It is shown that the solution of the main problem, expressed described by the equation.

$$
\{F[x]-\hat{O}(s), \quad F[x]\}_{L_{2}(z)}=0,
$$

is a Volterra polynomial of degree $\mathrm{N}$, having the form:

$$
F[x]=F_{N}[x]=\sum_{n=0}^{N} \int_{E_{+}^{n}} K_{n}\left(\tau_{1}, \ldots, \tau_{n}\right) \prod_{r=1}^{n} x\left(t-\tau_{r}\right) d v_{\tau} .
$$

We omit the intermediate calculations (for the purpose of brevity), and have the following result:

$$
\sum_{m=0}^{N} \int_{E_{+}^{m}} K_{m}\left(\xi_{1}, \ldots, \xi_{m}\right) \times m_{x}^{(n+m)}\left(\tau_{1}-\tau_{2}, \ldots, \tau_{1}-\tau_{n}, \tau_{1}-\xi_{1}, \ldots, \tau_{1}-\xi_{m}\right) d v_{\xi}=m_{s x}^{(1, n)}\left(\tau_{1}, \tau_{2}, \ldots, \tau_{n}\right)
$$


Let us briefly describe the main idea of the regularization method proposed in the second part.

Let us consider the operator equation $A x=y$, where A is a continuous non-linear bounded operator $x=x(t) ; y=y(t)$, are input and output signals, which are elements of the functional spaces E1 and E2, respectively, and the output $\mathrm{y}$ is given. It is required to find the function $x=x(t)$ from the equation $A x=y$. The solution of equations of this kind is known to be unstable, if the spaces E1 and E2 have different topologies. The solution of the original equation may not even exist in the functional space in which it is required to find it.

In particular, the problem of solving polynomial systems of integral equations determining an optimal nonlinear filter is ill-posed in the general case by HadamardTikhonov, since the exact solution $\bar{x}$ is unstable to the residuals of the right-hand side. Let us use a nonlinear regularizing functional having the following form:

$$
M_{\alpha}(x)=\varphi(A x-\bar{y})+\alpha \cdot f(x),
$$

where $\alpha>0$ is a so-called regularization parameter, $\varphi(\cdot)$ and $f(\cdot)$ are some $\Phi(L)$ functions, $\bar{y}$ is the image of exact solution $\bar{x}$.

Solving the equation $A^{*} \cdot A x+\alpha \cdot x=A^{*} y$ (where $A^{*}$ is an operator adjoint to the operator $A$ ), we obtain a family of regularized approximate solutions $\left\{x_{\alpha}\right\}$ for the initial equation $A x=y$ in the sense that for $\alpha \rightarrow 0$ the limit relation $\lim _{\alpha \rightarrow 0}\left\|\left(x_{\alpha}-\bar{x}\right) ; E_{1}\right\|=0$ is true, which is the end of optimal filtering process.

Let us suppose that the operator $A$ is subject to the condition $(\widetilde{A})$, if the following inequation is true:

$$
\left\|A x ; E_{2}\right\| \leq \psi\left(\left\|x ; E_{1}\right\|\right) \text { и } \frac{\left\|A^{\prime} x ; E_{2}\right\|}{\left\|f^{\prime} x ; E_{2}\right\|} \leq \gamma\left(\left\|x ; E_{1}\right\|\right),
$$

here, real-valued functions $\psi(\cdot)$ and $\chi(\cdot)$ are defined and strictly monotone in $\mathrm{R}^{+}$and, if one of the functions increases, then the other decreases.

Given that the point $x \in E_{1}, x \neq \theta_{E_{1}}\left(\theta_{E_{1}}\right.$ is the "zero" of the space $\left.E_{1}\right)$, realizes the local minimum of the functional $M_{\alpha}(x)$, and the A-operator is subject to the condition $(\tilde{A})$. Then the following optimal estimate holds for the parameter $\alpha$, where the latter is chosen according to the "exact residual" $\delta$, that is, the residual $\delta=\left\|A x-y_{\delta} ; E_{2}\right\|$ is given a priori:

$$
\alpha \leq \frac{\gamma\left[\psi^{-1}\left(\left\|y_{\delta} ; E_{2}\right\|-\delta\right)\right]}{\left\|\varphi^{\prime}(\delta) ; E_{2}\right\|} .
$$

\section{Conclusions}

Thus, we have proposed and considered some approaches to solving the problem of optimal synthesis of a nonlinear dynamical system in the form of a multidimensional nonlinear model of the system and to regularizing the solution obtained. 
The obtained equations received in our work would enable research on the dynamic characteristics of nonlinear oscillatory systems, taking into account the modern understanding of dynamic processes features.

\section{References}

1. V.M. Alekseev, V.M. Tikhomirov, S.V. Fomin, Optimal control (Fizmatlit, Moscow, 2007)

2. V.N. Volkova, A.A. Denisov, Theory of Systems (Higher School, Moscow, 2006)

3. M.A. Gayde.s, General theory of systems : systems and systems analysis (The Globe Press, Vinnytsa, 2005)

4. A.Kh. Gelig, I.E. Zuber, A.N. Churilov, Stability and stabilization of nonlinear systems (Publishing house of St. Petersburg State University, St. Petersburg, 2006)

5. A.A. Denisov, Modern problems of system analysis: Information bases (Publishing house of St. Petersburg State University, St. Petersburg, 2005)

6. V.V. Kachala, Foundations of the theory of systems and system analysis (Hot LineTelecom, Moscow, 2007)

7. V.G. Fetisov, V.I. Filippenko, O.I. Okhrimenko, Qualitative and quantitative methods of system analysis (FGBOU HPE «JURUES», Shakhty, 2011)

8. V.G. Fetisov, O.I. Okhrimenko, I.I. Panin, Selected chapters of modern control theory with applications (ISOiP (branch) of the DSTU, Shakhty, 2015) 